\title{
A CASE OF ATYPICAL LINDAU'S DISEASE
}

BY

\section{W. RUSSELL BRAIN, J. G. GREENFIELD, and D. W. C. NORTHFIELD}

The case to be described is unusual in presenting most of the changes of Lindau's disease without any cerebellar tumour. In addition there is evidence of a hereditary tendency to the disease, as four relations of the patient suffered from progressive visual defect, and one other had an intracranial tumour.

The patient herself had an hæmangioblastoma in one retina and two in the other, an hæmangioblastoma of the spinal cord with extensive syringomyelia and cystic pancreas and kidneys.

\section{History of illness}

The patient, a female aged 39 years, was referred by Dr. W. A. Steel, Medical Superintendent of Hillingdon Hospital, and was admitted to the London Hospital on the 25th August, 1939, and later transferred to Chase Farm E.M.S. Hospital. Fifteen years previously she began to lose the sight of the right eye. Two years later, Mr. Frank Juler examined the patient and found the following condition : "In each eye the superior temporal branches of the central artery and vein show enormous dilatation, and appear to terminate in rounded prominent whitish masses, of which one is visible in the upper temporal periphery of the right eye, one in the upper periphery of the left eye and another a short distance external to the macula in the left eye. At the macula of the right eye there is a large deposit of yellow-white substance with a fairly sharp outline and a prominent circular black patch in its centre, probably an organized hæmorrhage. In the temporal periphery of the left eye there is some retinal detachment, and pre-retinal striae. R.V. $=6 / 60$. L.V. $=6 / 5$." Drawings of the fundi were made by Messrs. Theodore Hamblin Limited at that time, and are reproduced (Figs. $1 a$ and $b$ ). Mr. Juler examined the patient again in February, 1929, and found : "The condition has progressed and the right eye has become cataractous, with detachment. Left eye-vitreous opacity is present. The superior temporal vascular dilatation is not so marked as in 1926, but the inferior temporal vessels are larger. There is more fibrosis over the fundus which obscures detail. The mass near the macula is visible but less obvious. There is also an indefinite mass in the upper periphery, evidently the remains of the swelling seen in 1926. There is peripheral detachment of the upper and lower temporal retina. The visual field shows a marked infero-nasal loss. L.V. $=6 / 18$ partly.", Dr. Wilfred Harris found no abnormality of the nervous system, and an X-ray examination of the skull was normal. Subsequently, in 1929, the right $\stackrel{\mathbb{D}^{\circ}}{2}$ eye was removed on account of pain said to be due to retinal angioma. The sight of the left eye con-tinued to deteriorate until she could only perceive: light.

Three years ago a feeling of weakness developed in the toes of the left foot, there was intermittent stiffness of the left leg, and the foot would " jump? off the ground." There was a feeling as though the - ' shoe were too tight. One year ago the left legn' became weak and clumsy and there was pain in the thigh on sitting. Nine months ago a "tight"을 feeling developed in the toes of the right foot, but the right leg has been weak for only three or fourc months. She could walk only with help. She had occasional discomfort at the left subcostal margin an occasional crushed feeling in the ulnar fingers of the left hand, and for a few weeks pain in the lefte side of the neck, the left shoulder and left arm.ढ There was no girdle sensation and no sphinctery disturbance. A number of her relatives had had illnesses which were highly suggestive of angioma (Fig. 2).

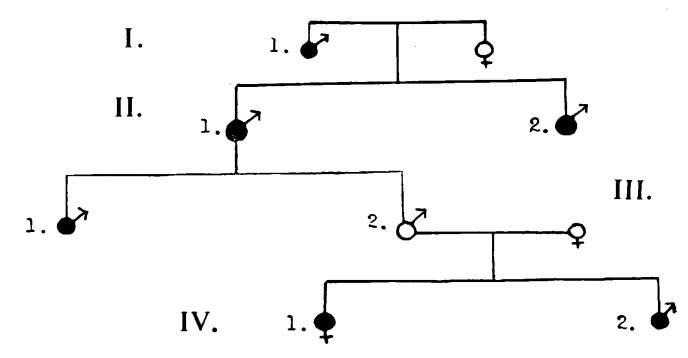

I. 1. Blind

FIG. 2.

II. 1. Blind.

II. 2. Became blind and lost sense of taste.

III. 1. Had an intracranial tumour.

III. 2. Had no nervous symptoms and died of enteritis $\mathrm{N}$ IV. 1. The patient.

IV. 2. Stated to have " retinitis."

Examination (17th October, 1939). No cutaneous $\mathrm{c}^{\mathrm{N}}$ tumours or pigmentation were found and the pulmonary, cardiovascular and alimentary systemse were normal. Blood pressure $130 / 80 \mathrm{~mm}$. $\mathrm{Hg} . \bar{\Phi}$ Mentality, speech and articulation were normal: Right eye removed. Left fundus was invisible -5 owing to cataract. Movements of the left eye weres full, but there was nystagmus on fixation in alf directions. The cranial nerves were otherwise normal. The upper limbs were normal. The lower limbs showed moderate muscular wasting andô 


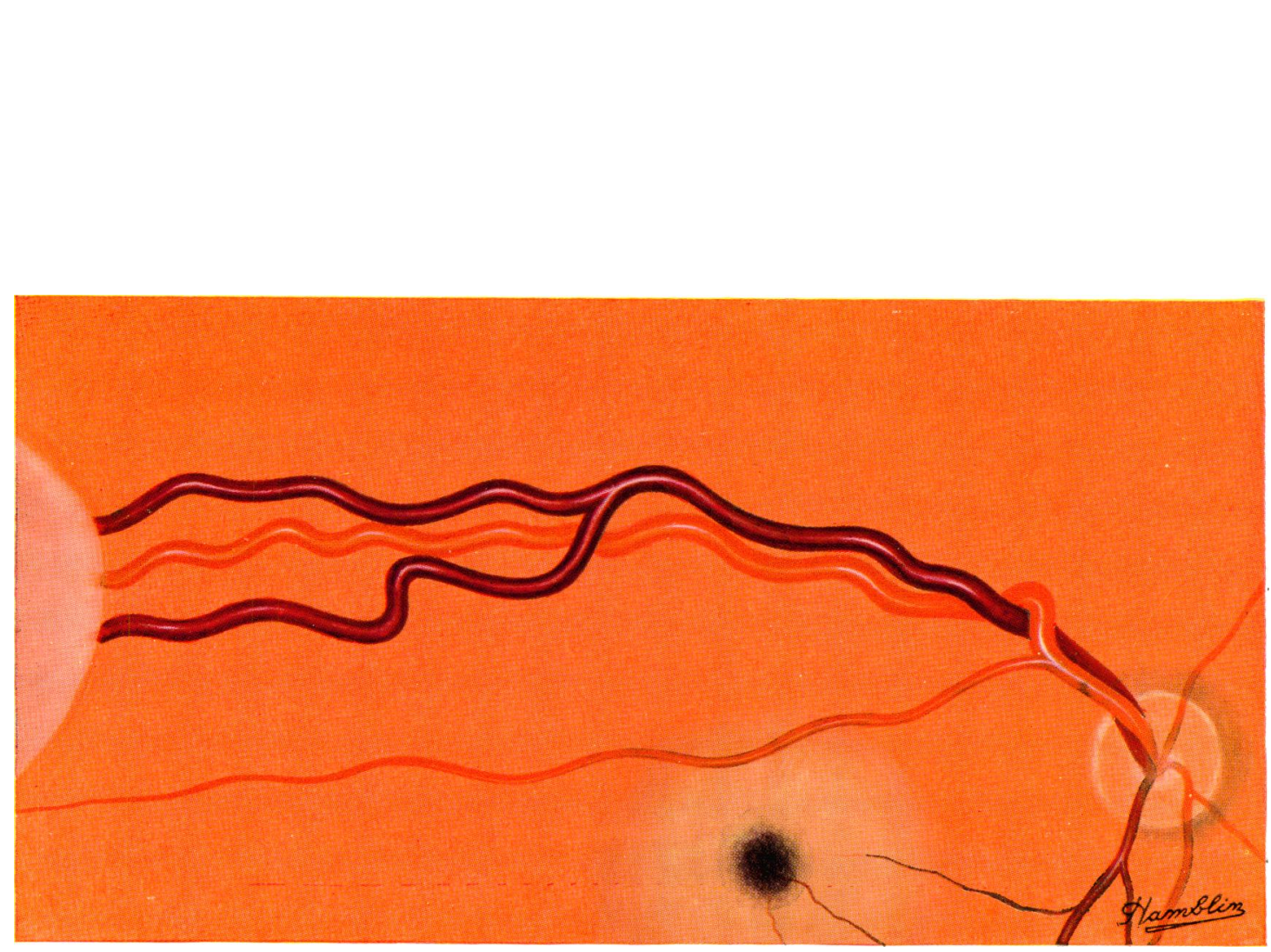

Fig. 1a.-Fundus of right eye.

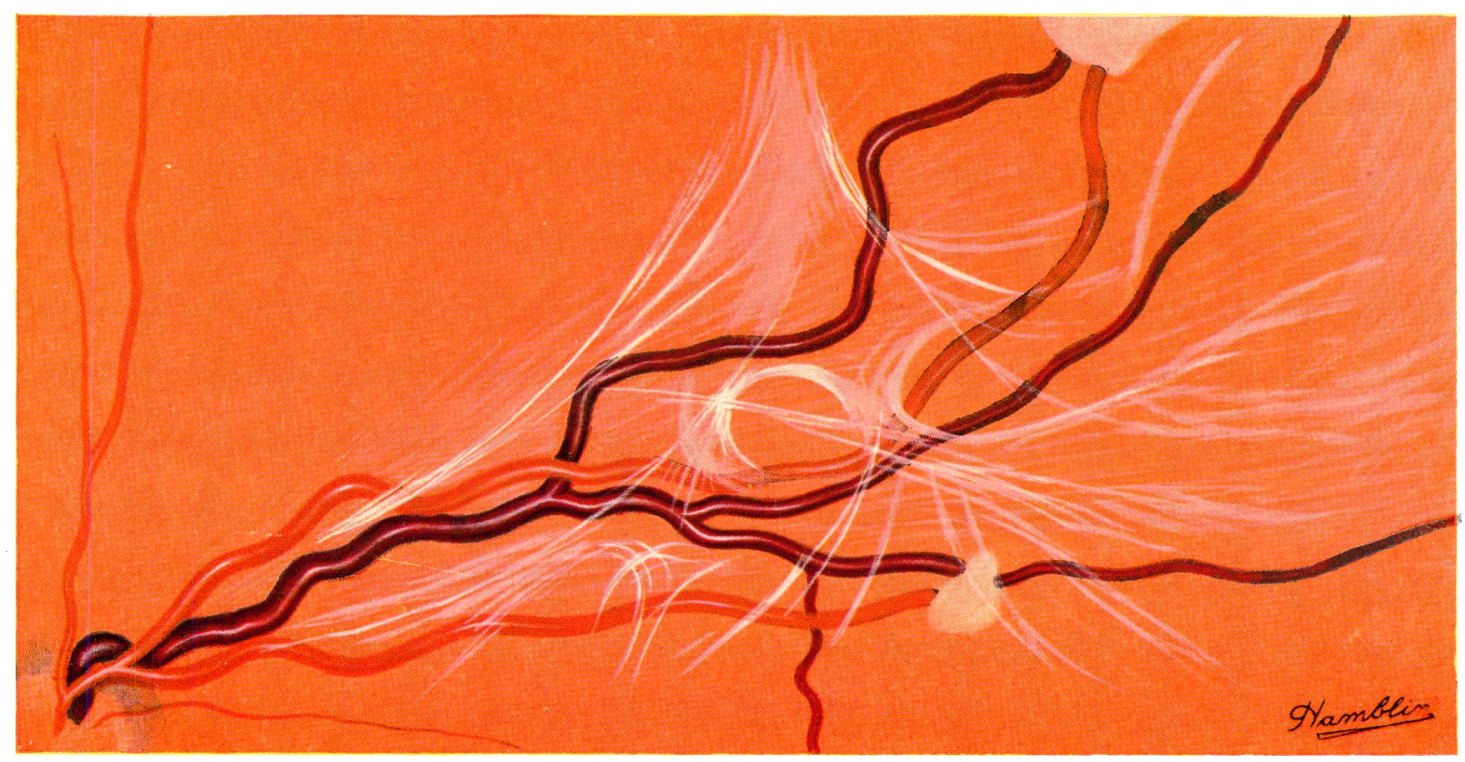

Fig. 1b.-Fundus of left eye. 
spastic weakness greater on the left side. Bilateral ankle clonus and extensor plantar responses were obtained. The abdominal reflexes were absent. Relative cutaneous anæsthesia and analgesia were found below the eighth thoracic segments on both sides with sacral sparing, but the upper limit of this impaired sensation was ill-defined. Slightly impaired thermal recognition was found over the front of each lower limb. There was considerable impairment of postural sense in the toes, and of vibration sense at the ankles; scoliosis was present, to the right in the thoracic and to the left in the lumbar region. Lumbar cerebrospinal fluid, resting pressure $120 \mathrm{~mm}$. rising slowly and jerkily to $200 \mathrm{~mm}$. on jugular compression and rising more quickly to $280 \mathrm{~mm}$. on abdominal compression, falling quickly to $180 \mathrm{~mm}$. and then slowly and jerkily to $140 \mathrm{~mm}$. : pale yellow, slightly turbid fluid, containing 11 lymphocytes per c.m. and $1800 \mathrm{mgms}$. of protein per 100 ccs.

$X$-rays of the vertebral column revealed no abnormality; after lumbar injection of lipiodol, Dr. Vilvandré reported : " Main bulk of the lipiodol remains low down in the spine, about the third and fourth lumbar vertebra, but the lipiodol is split up and appears as globules as far up as the eleventh thoracic vertebra, where it seems to remain for 20 minutes. On further tilting (head down), after another 20 minutes the upper level of the lipiodol globules is unaltered; there seems to be stoppage at the eleventh thoracic vertebra, but throughout the lipiodol appears broken up ; there is no obvious evidence of complete narrowing of the canal, but general breaking up of the lipiodol." After cisternal injection, lipiodol rapidly descended the spinal theca without delay at any level.

A compression paraplegia was clearly present, but the level was indefinite, since the clinical and radiological findings did not coincide (thoracic sixth and thoracic eleventh vertebrae respectively). An angioblastoma seemed the most likely lesion, in view of the family and personal history, and of the high protein content of the cerebrospinal fluid with only a relative degree of subarachnoid block. One of us (D. W. C. N.) favoured a meningioma as the most likely extramedullary lesion to give such a slow progression and relatively severe incidence of posterior column loss.

On the 19th October, 1939, laminectomy of T 5-6 was undertaken but operation had to be abandoned after the bone had been removed on account of respiratory difficulties. Four days later further operation was undertaken, and as the exposed dura did not pulsate, further laminae in an upward direction were also removed. The spinal cord was very swollen and covered with a great number of adventitious vessels. No tumour was detected. An aspirating needle was inserted into the cord and 6 ccs. of clear faintly straw coloured fluid withdrawn. This was followed by shrinking of the cord and commencement of well marked pulsation. A small fragment of the wall of this intramedullary cavity was removed for biopsy and the wound closed. Histological examination showed this tissue to be the wall of a syringomyelic cavity.

Recovery from these operations was at first satisfactory. A week later, there was complaint of numbness, tingling and flushing of the face, and numbness of the hands so that she could not knit. Examination revealed no change in the state of the cranial nerves ; but in the upper limbs there was some increase of the tendon jerks of the right arm, ataxia, and slight impairment of postural sensibility of the little fingers. There was marked exaggeration of the signs of thoracic cord compression, for now there was virtually complete sensori-motor paraplegia below the tenth thoracic segment, and it was fully appreciated that a spinal tumour was present at a level lower than the laminectomy. Further operation was postponed owing to the

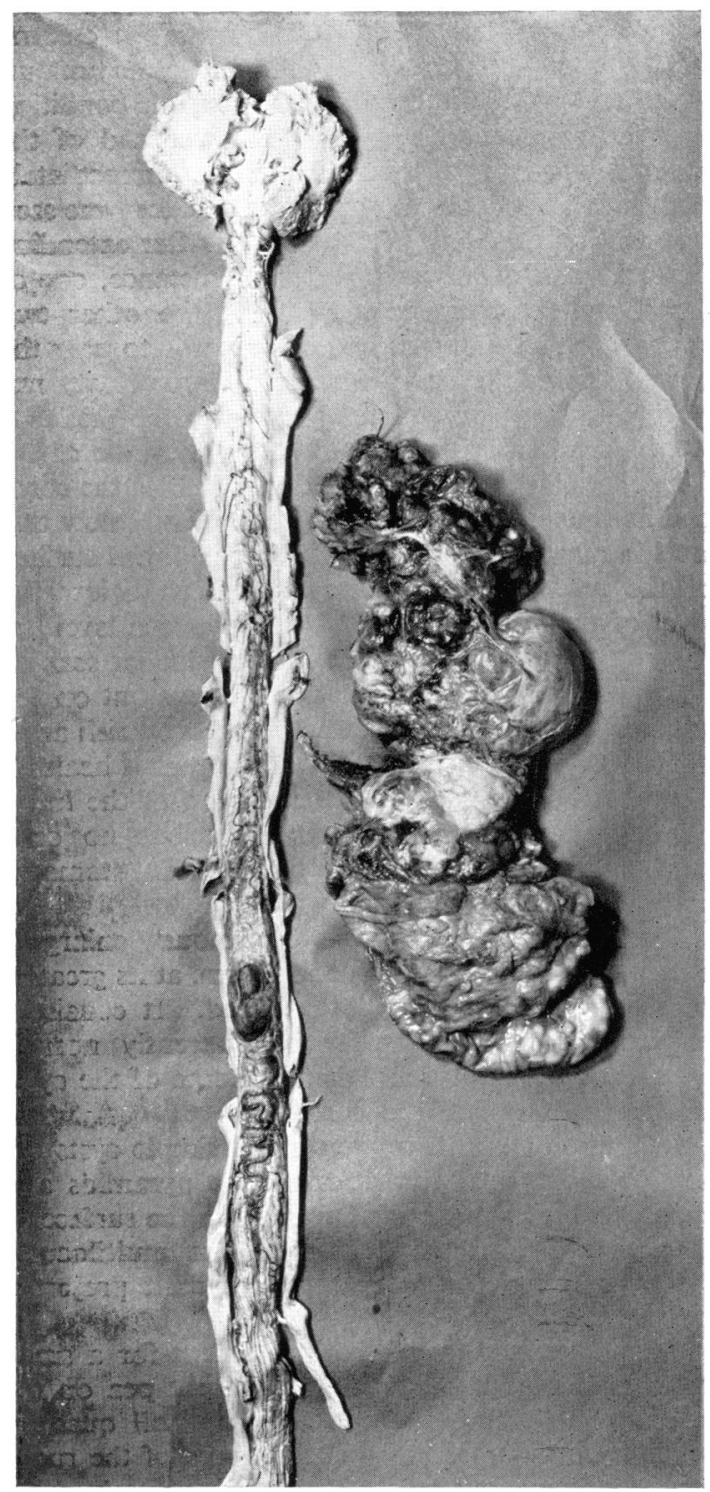

Fig. 3.-Spinal cord and pancreas. 
patient's frailty. A few days later she developed signs indicating further spread of paralysis in the cord, i.e. greater ataxia, gross posterior column loss, palatal paralysis (motor and sensory), cough and cyanosis-and she died on the 6th November, 1939.

Post mortem examination.-This was made on the 6th November, 1939, less than twelve hours after death. The brain showed no abnormality except atrophy of the right optic nerve.

The spinal cord (Fig. 3) had been incised at operation at the lower end of the first thoracic segment. The whole cord was wider than normal, due to the presence in it of syringomyelic cavities. In the lower thoracic region there was an oval, orange-red tumour embedded in the dorsal surface of the cord at the level of the ninth and tenth thoracic segments, the lower part of the ninth and the whole of the tenth segment being involved. It measured $3 \times 1.6 \mathrm{c} . \mathrm{m}$. The vessels on the dorsal surface below it were dilated and tortuous. The column of Goll was degenerated and grey above the tumour.

On section of the cord a syringomyelic condition was found extending up to the lower end of the medulla and into the conus terminalis. Immediately above and below the tumour three cavities were seen in transverse sections of the cord. After extending upwards and downwards for some distance, one of these cavities disappeared, leaving the other two cavities to run upwards and downwards to near the extremities of the cord, whence a single cavity ran down into the conus and upwards to the medulla. At the level of the pyramidal decussation the cavity filled a large part of the right dorsal half of the cord, and as the cord had been cut across just below this level in removing it from the body the dorsal surface of the medulla had become depressed below the level of the left side (Fig. 4). Above this level the cavity rapidly narrowed and was no longer seen at the lower end of the calamus scriptorius but on the left side here was a small discoloured brownish area.

The thyroid, heart, and aorta were healthy. There was some hypostatic congestion of the lungs especially in the right lower lobe, but no consolidation was present. The liver, spleen, stomach, and intestines were normal.

The pancreas (Fig. 3) was enormously enlarged, measuring $27 \mathrm{~cm}$. in length and $10 \mathrm{~cm}$. at its greatest width, which was towards the head. It consisted of very numerous cysts with apparently normal pancreatic tissue between them. Some of the cysts contained thin, dark red fluid.

In both kidneys there were small simple cysts the size of a pea at the junction of the pyramids and cortex and a few were also present on the surface of the organ. In the right kidney one multilocular cyst was found involving a papilla as it projected into the pelvis.

The suprarenals were normal except for a small accessory suprarenal the size of a split pea on the left side. The bladder contained a small quantity of milky purulent fluid. The surfaces of the rugae were inflamed, but the wall was otherwise healthy. The ovaries and uterus were normal. The right
Fallopian tube was adherent to the mesentery in the region of the appendix.

The back of the left eyeball was removed from a few millimetres behind the ciliary body. The retina was seen to be thrown forwards except where it was attached to the disc by a rather broad pedicle, the front part of the retina being spread out flat behind the lens. The arrangement resembled an open umbrella of which the handle was represented by the pedicle. The retina was firmer than normal. Round the disc and overlying the choroid there was a whitish zone of fibrosis which was partly ossified. No angiomatous malformation could be seen on that part of the retina which remained attached to the choroid anterior to the plane of section.

Microscopical examination.-The posterior half of the left eye was sectioned in the antero-posterior plane. It was then seen that the pedicle already described was made up of thinned out retina, the two layers of which separated gradually from one another as they passed forwards and they then curved outwards to reach the lateral wall of the eyeball. Over the funnel-shaped cavity which thus presented forward, there was stretched a thin layer of collagenous connective tissue, lined on its posterior surface by a thin layer of endothelium. This became attached laterally to the anterior surface of the retina. It probably represented a greatly thickened anterior limiting membrane. The retina itself was everywhere very degenerated with disappearance of the outer granular layer and a great increase of neuroglial fibres, but the ganglion cells and the inner granular layer could be distinguished everywhere except in the region of the tumours.

Two definite small angioblastomas were found in the retina. One lay on the outer surface of the retina close to the disc. The two layers of retina were here joined together by thin strands of neuroglial tissue. This tumour was lentiform, measuring $0.4 \mathrm{~mm}$. in thickness and $2.6 \mathrm{~mm}$. in length. It consisted of a tangle of capillary vessels and contained also many larger thin-walled blood vessels about $30 \mu$ in diameter. Many of the endothelial cells forming the walls of the capillaries were swollen with rather granular cytoplasm. No neuroglial elements were seen within the tumour, although there was considerable overgrowth of neuroglial fibres round the tumour, and some fibres passed into its outermost zone.

The other tumour which was exactly similar in structure, lay just external to the point of junction between the thickened anterior limiting membrane and the retina. This was of the same thickness as the tumour nearer to the disc but did not extend in the plane of section for more than $1 \mathrm{~mm}$. laterally. Some retinal pigment had migrated into this tumour. It was covered on its anterior surface by the thickened anterior limiting membrane, and several irregular whorls of dense collagen spread from this into the anterior layers of the tumour. Several fairly large cysts lay in the retina lateral to it.

A thin layer of bone lay over the choroid pos- 


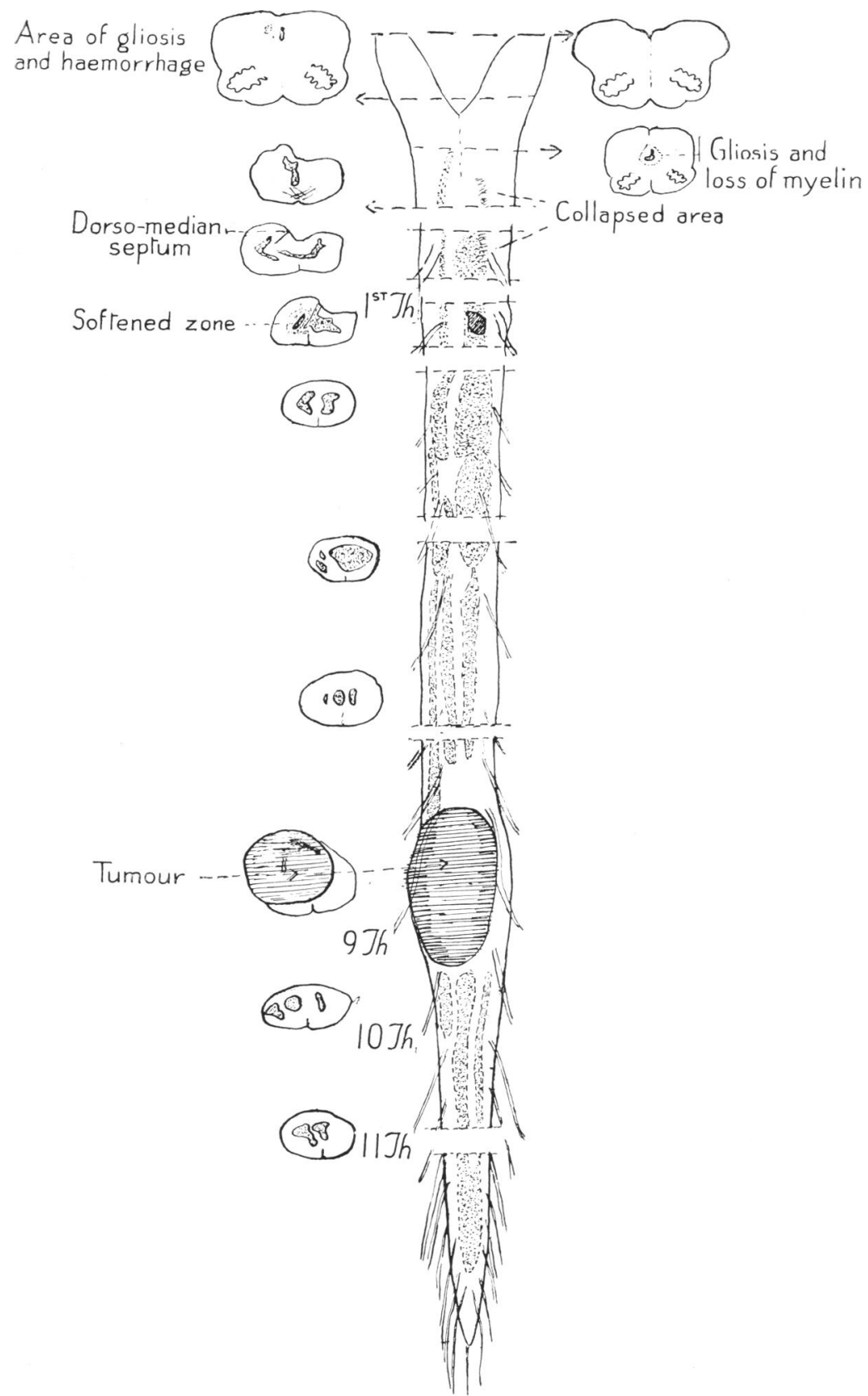

FIG. 4.

teriorly, forming a fairly wide zone round the disc. The optic nerve showed no evidence of degeneration.

The spinal tumour was a fairly typical hæmangioblastoma, the only abnormal feature being the presence of occasional very large irregularly shaped nuclei, some of which lay in large vacuolated cell bodies. The amount of collagen varied greatly in different parts of the tumour and was in inverse proportion to the degree of cellularity. There were fewer foamy cells than in many hæmangioblastomas of the central nervous system. With hæmatoxylin and eosin and with Masson's trichromic stain a few medium sized cells filled with oxyphil granules were seen in some areas. The syringomyelic cavities were lined by a fairly thick wall of astrocytes with thin fibres arranged for the most part concentrically to the cavity. The zone of gliosis varied little in thickness but was thinnest in the cervical region where it was about $250 \mu$, and thickest in the lumbar region where it reached $400 \mu$. At the level of the operation there was a narrow zone of softening anterior and lateral to the zone of gliosis surrounding the cavity which had been opened. This had been partially organized but still contained fat granule cells. Some rarefaction of the tissues in the same relation to this cavity could be traced a few 
segments higher but none was seen at the level of the first cervical segment. The zone of softening at the first thoracic level passed through the position of the central canal, and it could not be identified here with certainty. At the other levels sectioned it lay ventral and internal to the cavities and was represented only by an irregular clump of ependymal cells. No ependymal lining was found in any of the cavities.

In the kidneys the cysts appeared to be lined by flattened epithelium, but this was rather broken in the sections. Round some there was an excess of small congested vessels which became gradually less numerous on passing outwards into the normal renal tissue. No hæmangiomas or vascular malformations apart from these could be seen in relation to any cyst. In one large cyst there was a collecticn of irregularly shaped cells with medium sized oval nuclei and irregularly cuboidal or elongated cell body. Some of these were packed side by side and resembled the desquammated epithelium of a collecting tubule. An occasional multinucleated cell was seen among them. Round this cyst there was a zone of connective tissue containing many foamy cells.

In the pancreas the cysts appeared to be formed by dilatation of ducts of all sizes. Many were surrounded by very thick connective tissue walls, containing large vascular channels. Apart from the cysts and dilated ducts there was a great excess of interstitial collagen in the pancreas which broke up the parenchymatous tissue into small groups of acini. No other abnormality was found in the pancreatic tissue.

\section{Noteworthy clinical features}

Until operation revealed the syringomyelia, no explanation was forthcoming for the pains on the left side of the neck and in the left upper limb, nor for the nystagmus. These symptoms were the only evidence of syringomyelia above the eighth thoracic segment, which was the upper level of the relative cutaneous sensory loss. The level of the first operation was determined by this clinical finding, as radiograpliy with lipiodol gave little help. It is probable that the tumour was immediately below the site of this exploration, but no search was made in a downward direction owing to the absence of pulsation of the dura, due to the distension of the cord.

The rapid development of paralysis of the cervical cord and of the bulb after drainage of the cavity in the spinal cord may have been due to some vascular disturbance of the surrounding tissues. Following operative interference above the level of the tumour, there was also aggravation of the compression paraplegia. This type of reaction is not infrequent after lumbar puncture or after the cisternal injection of lipiodol, and is generally ascribed to displacement and wedging of the tumour, as a result of alterations in its water ked. This may have occurred in this instance, although the tumour was so securely anchored to the spinal cord as to render its displace- ment very unlikely. More probably, a vascular disturbance accounted for these symptoms as well as for those at the higher level

\section{Pathological discussion}

The case described is one of Lindau's disease in its most complete form, with one important exception ; the tumours and accompanying cyst formation in the central nervous system were in the spinal cord, and no tumour was found in the cerebellum. It is also unusual in the fact that two hæmangioblastomas were present in the retina of the left eye.

It must be remembered that Lindau was led to discover the syndrome to which his name has been given, by a study of cerebellar cysts, and therefore perhaps too much emphasis has been laid on the cerebellar incidence of the tumours. It seems probable that hæmangioblastomas of the spinal cord are less rare than was at one time thought, though comparatively few have been put on record. Kernohan, Woltman, and Adson found four hæmangioblastomas in the spinal cord, and three in the filum terminale and cauda equina among 67 primary tumours in these situations. These figures show an unusually high incidence of spinal hæmangioblastomas. In the cases examined at the National Hospital, Queen Square, during the period of twenty years from 1920 to 1939 , only two hæmangioblastomas were found in relation to the spinal cord, both being extra-medullary. In this material there were 41 cases of glioma of the spinal cord, 7 of these being more or less symptomless tumours in cases of generalized neurofibromatosis. During the same period there were 32 cases of cerebellar hæmangioblastomas, among 164 primary tumours of the cerebellum and fourth ventricle, the great majority of which arose from nervous structures, i.e. gliomas, ependymal and choroid plexus tumours and neuroblastomas or ganglioneuromas. According to these figures hæmangioblastomas form a smaller proportion of primary tumours in the spinal cord and cauda equina than in the cerebellum and fourth ventricle. In the cerebral hemispheres their incidence is still lower and their relationship to primary tumours of the hemispheres very small indeed.

The literature of the last few years has brought to light a number of cases of multiple haemangioblastomas of the central nervous system, and in the great majority of these one or more tumours has been present in the spinal cord or its meninges. Our case differs from these only in the absence of any tumour in the cerebellum or fourth ventricle. Such cases have been reported by Schubach (1927), Kernohan, Woltman and Adson (1931), Wolf and Wilens (1934), Davison, Brock and Dyke (1936), Konig and Schœn (1939) and Craig, Wagener and Kernohan (1941). In 5 of these cases there was a hæmangioblastoma in the cerebellum and in the other, that of Konig and Schoen, as well as in that of Craig, Wagener and Kernohan, similar tumours in the fourth ventricle. In neither of these cases were retinal tumours found, but in three of the 
others they were present in both eyes, and in the fourth on one side only. In all these six cases there was syringomyelia of greater or less extent in association with the spinal tumours. A cystic pancreas was present in all. The kidneys were described as cystic in all, except the case of Wolf and Wilens, and tumours described as adenomas, hypernephromas or carcinomas were present in all except the case of Konig and Schoen. A definite hereditary tendency was found in the cases of Konig and Schoen and of Craig, Wagener and Kernohan only.

Most of these authors comment on the relationship of the syringomyelia to the tumours. It appears in every case to have been a true syringomyelia in that it made only fortuitous connections with the central canal and over the greater part of its extent was separate from it. The consensus of opinion is that the syringomyelia resulted from a separate developmental abnormality and was not caused, as the cerebellar cysts appear to be, by transudation of plasma into the nervous tissue from the tumour. In our case the extension of the cavities up to the medulla, where they terminated in the grey matter round the central canal, that is in the same position as the cavities of idiopathic syringobulbia, points in the same direction.

We are greatly indebted to Mr. Frank Juler for his permission to use his clinical notes and the drawings of the fundi.

\section{REFERENCES.}

Craig, W. M., Wagener, H. P., and Kernohan, J. W. (1941). Arch. Neurol. Psychiat. (Chicago), 46, 36.

Davison, C., Brock, S., and Dyke, C. G., (1936). Bull. neurol. Inst. N. Y., 5, 72.

Kernohan, J. W., Woltman, H. W., and Adson A. W. (1931). Arch. Neurol. Psychiat. (Chicago), 25, 679.

König, E., and Schoen, H. (1939), Beitr. Klin. Chir., 170, 239.

Schuback, A. (1927), Z. ges. Neurol. Psychiat. (Chicago), 110,359 .

Wolf, A., and Wilens, S. L. (1934), Amer. J. Path., 10, 545 . 